Case Report

\title{
Conventional Cementless Total Hip Arthroplasty for Hip Osteoarthritis after Distal Trochanteric Transfer: A Report of Two Cases
}

\author{
Hirohito Hirata, Motoki Sonohata $(\mathbb{D}$, Akira Hashimoto, Sakumo Kii, Takema Nakashima, \\ Masaya Ueno, Shuichi Eto, Shunsuke Kawano, and Masaaki Mawatari
}

Department of Orthopaedic Surgery, Faculty of Medicine, Saga University, Saga 849-8501, Japan

Correspondence should be addressed to Motoki Sonohata; epc9719@yahoo.co.jp

Received 22 May 2020; Revised 13 July 2020; Accepted 31 July 2020; Published 11 August 2020

Academic Editor: Eyal Itshayek

Copyright (C) 2020 Hirohito Hirata et al. This is an open access article distributed under the Creative Commons Attribution License, which permits unrestricted use, distribution, and reproduction in any medium, provided the original work is properly cited.

\begin{abstract}
Distal trochanteric transfer (DTT) has been widely performed to treat developmental dysplasia of the hip or Perthes disease. Total hip arthroplasty (THA) following DTT in patients with hip osteoarthritis is one of the most challenging procedures for hip surgeons, because great care must be taken regarding anatomical abnormalities of the greater trochanter and the soft tissue attached to the greater trochanter. To the best of our knowledge, there are no reports on THA after DTT. We herein report two cases of patients who underwent THA post DTT using cementless components. After THA, both patients developed abduction temporary contraction because of leg length extension and gluteus medius hypertension. However, in both cases, the contraction was reversible within two months and the final clinical result was good. Therefore, THA can be considered an effective and safe choice for treating osteoarthritis after DTT.
\end{abstract}

\section{Introduction}

Deformities of the proximal femur such as coxa vara and high-standing greater trochanter are known to occur because of conditions such as developmental dysplasia of the hip and Perthes disease [1]. Leg length discrepancy (LLD) and shortening of the abductor musculature can cause a reduction in the abductor lever arm and Trendelenburg gait [2-6]. Distal trochanteric transfer (DTT) has been widely performed to increase tension of the gluteal muscles and hip abductors $[7,8]$. Although good results are reported with the procedure, studies have also shown that DTT does not prevent or lower the risk of the development of osteoarthritis of the hip [6,9, 10]. In a previous study, $40 \%$ of patients who underwent DTT subsequently required hip arthroplasty for osteoarthritis [6]. THA for patients with hip osteoarthritis following osteotomy of the proximal femur, including DTT, is one of the most challenging procedures for hip surgeons $[11,12]$.
In particular, surgeons must take great care regarding anatomical abnormalities of the greater trochanter and soft tissue attached to the greater trochanter. However, there have been no reports regarding a focus on performing total hip arthroplasty (THA) after DTT. Therefore, it is unclear if conventional THA can yield satisfactory results in such a situation. Here, we report the results of conventional cementless THA after DTT.

This study protocol adhered to the ethical guidelines of the 1975 Declaration of Helsinki. The study was approved by our institutional review board. Both patients were informed that their cases would be submitted for publication, and they provided informed consent for the same.

\section{Case Presentation}

2.1. Case 1. A 56-year-old woman (height $156 \mathrm{~cm}$, weight $46 \mathrm{~kg}$ ) presented to our hospital with right hip pain, limited 


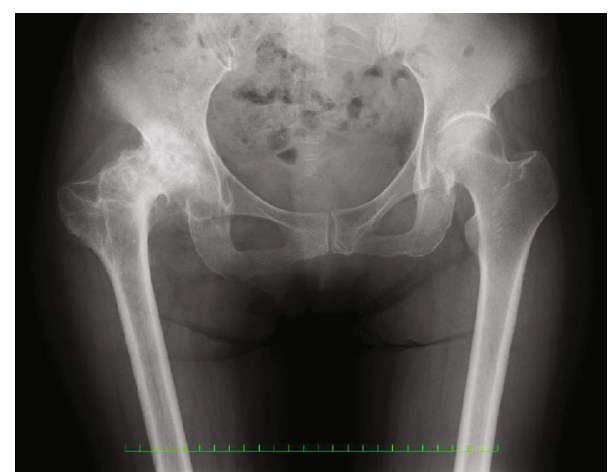

FIGURE 1: Radiographs of Case 1 before total hip arthroplasty.

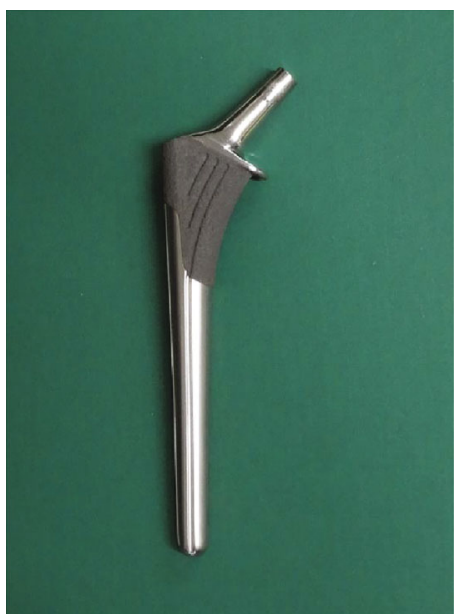

Figure 2: PerFix-HA femoral component used for implantation.

range of motion, and limping. Trendelenburg gait was observed. Her medical history included treatment for developmental dysplasia of the hip (DDH) with a cast when she was an infant. She developed hip joint pain and underwent DTT at the age of 23 years. Her hip pain improved after the surgery; however, she started to feel pain again when she was 40 years old.

Radiographs showed severe osteoarthritis of her right hip joint and the greater trochanter, which had moved to the level of the lesser trochanter (Figure 1). On anteroposterior radiography, a line was drawn through the teardrops and vertical from the teardrops. The vertical distance of the proximal lessor trochanter tip to this reference line was measured. The LLD was $36 \mathrm{~mm}$.

Conventional cementless THA was performed through a posterolateral approach with the patient in the lateral position under spinal anesthesia; the femoral component (PerFix-HA collared STD stem, neck-shaft angle $135^{\circ}$, Kyocera, Kyoto, Japan) (Figure 2) was used with a zirconia ball and an AMS-HA acetabular shell with a cross-linked ultra-highmolecular-weight polyethylene elevated liner (Kyocera, Kyoto, Japan).

Intraoperatively, as scar tissue augmentation was present due to the previous surgery, the surgeon paid close attention to identify the gluteus medius and minimus muscles and

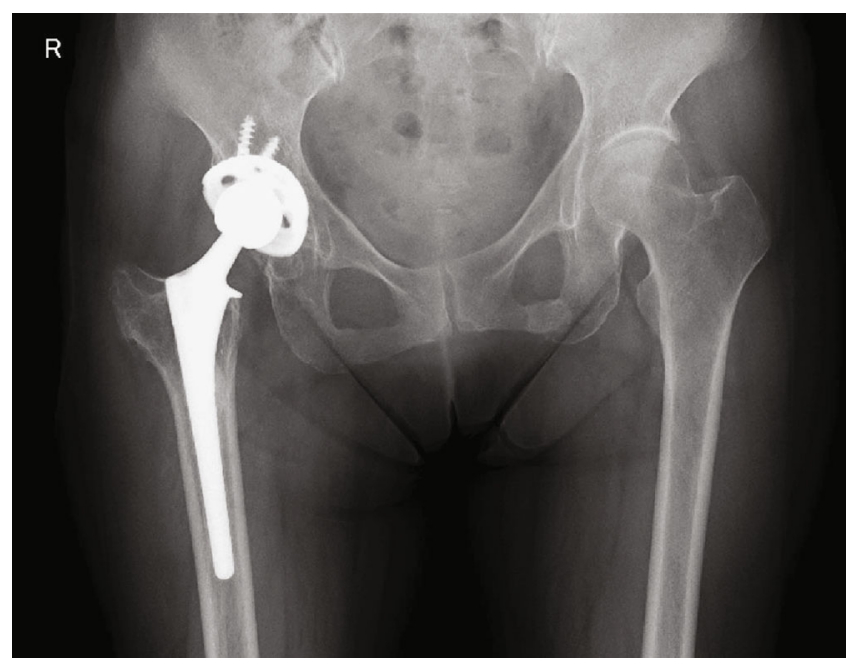

FIgURe 3: Radiographs of Case 1 last follow-up (7 years) after total hip arthroplasty.

prevent damage to both muscles due to hypertension during the reduction after implant placement. The total operation time was $59 \mathrm{~min}$, and the total blood loss was $675 \mathrm{~g}$ (intra and postoperative blood loss). The leg lengthening achieved was $18 \mathrm{~mm}$, and $18 \mathrm{~mm}$ of the LLD persisted.

The patient was allowed to stand and walk with full weight bearing after drain removal, 2 days after the operation. The pain improved remarkably; however, she presented with LLD due to an abduction contraction following extension of the leg length. However, the abduction contraction improved within two months under physical therapy. No other complications were observed, including dislocation, neurovascular abnormality, or infection. Final radiographs at 7 years after THA did not show subsidence of the stem or any type of loosening around the cup or stem (Figure 3).

We used the Japanese Orthopedic Association (JOA) hip score to evaluate the hip joint [13]. The JOA hip score includes four categories that amount to a total of 100 points as follows: pain (40 points), range of motion (20 points), walking (20 points), and activities of daily living (20 points). The preoperative JOA hip score was 46 points, which improved to 96 points at 7 years after surgery. Trendelenburg gait had improved.

2.2. Case 2. A 50-year-old woman (height $155 \mathrm{~cm}$, weight $60 \mathrm{~kg}$ ) first presented to our hospital with left hip pain, limited range of motion, and limping. Trendelenburg gait was observed. She did not have a history of treatment for DDH. She developed hip joint pain and underwent right DTT at the age of 20 years. Her hip pain had improved after the surgery; however, she started to feel pain again at 2 years after DDT. She underwent right-sided THA at another hospital at the age of 47 years.

Radiographs showed severe osteoarthritis of her left hip joint and the left greater trochanter, which had moved to the level of the lesser trochanter. The past THA on the right hip joint was visible as well (Figure 4). The LLD was $55 \mathrm{~mm}$. 


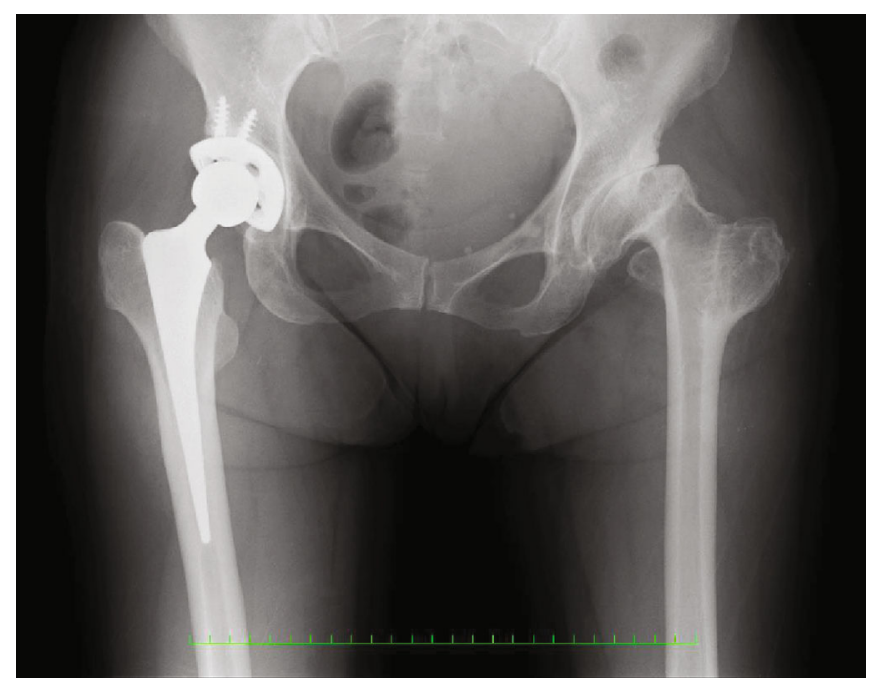

Figure 4: Radiographs of Case 2 before left total hip arthroplasty.

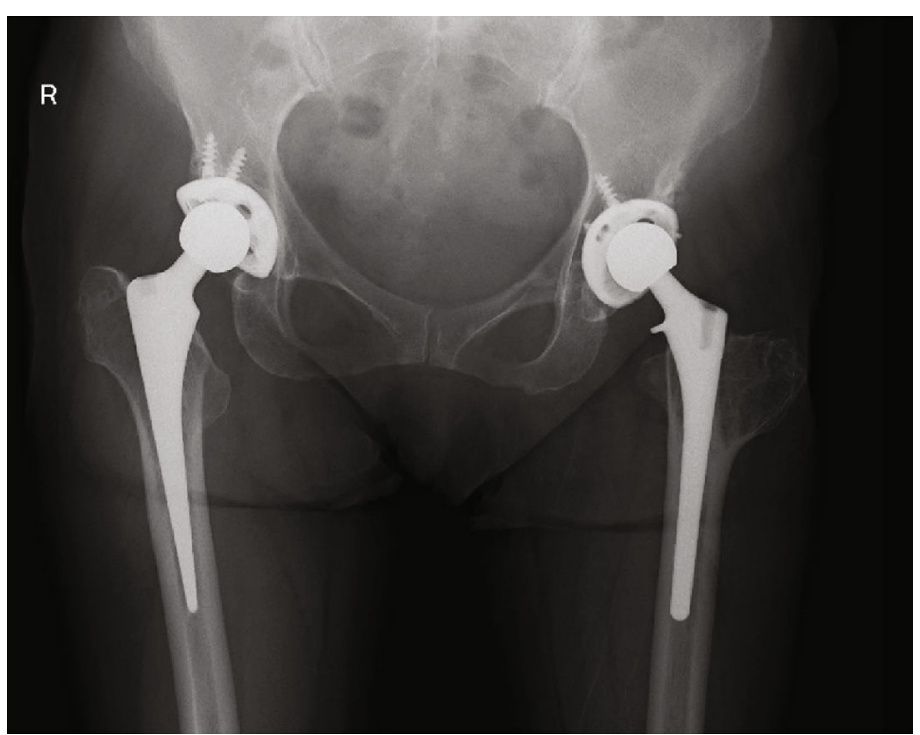

Figure 5: Radiographs of Case 2 at their last follow-up (11 years) after total hip arthroplasty.

The right THA was performed at the age of 47 , and the left THA was performed at the age of 50. The THA procedures comprised conventional cementless THA in the same way as in Case 1 (anesthesia, surgical approach, implants, and rehabilitation).

Intraoperatively, the surgeon had to pay close attention to identify the gluteus medius and minimus muscles and prevent damage to the same muscle due to hypertension during the reduction after implant placement, similar to the circumstances of case 1 . The operation time was $60 \mathrm{~min}$, and the total blood loss was $488 \mathrm{~g}$ (intra and postoperative blood loss). The leg lengthening was $27 \mathrm{~mm}$, and $28 \mathrm{~mm}$ of the LLD persisted.

As in Case 1, the pain improved remarkably; however, the patient presented with LLD due to an abduction contraction following extension of the leg length. The abduction contraction improved within two months under physical therapy. No other complications, such as dislocation, neuro- vascular abnormality, or infection, were observed. Final radiographs at 11 years after left THA did not show subsidence of the stem or any type of loosening around the cup or stem (Figure 5). The preoperative JOA hip score was 39 points, which improved to 71 points at 11 years after surgery. Trendelenburg gait had improved.

\section{Discussion}

Both the cases showed good results of THA following DTT. However, postoperative temporary abduction contracture was caused by the leg extension in both cases. DTT is known to be associated with an increase in hip abductor tension [11, 12]; unfortunately, this often means that DTT needs additional THA $[6,9,10]$. It is well-known that THA following osteotomy of the proximal femur involves many technical difficulties as compared to primary THA $[11,12]$. There are some reports on THA for hip osteoarthritis following 
proximal femoral valgus osteotomy, proximal femoral varus osteotomy, Schanz osteotomy, and anterior rotational osteotomy of the femoral head $[11,12,14,15]$; however, few reports exist on THA following DTT.

Surgeons should be aware of the following 2 surgical pitfalls. First, maximum attention should be paid when exposing the joint capsule to avoid muscle damage; the gluteus medius and minimus muscles are present distal to the joint capsule and are therefore vulnerable to damage. As both the gluteus medius and minimus muscles may have been damaged by previous surgery, manipulation should be done with caution. Damage to these muscles can lead to formation of a limp after THA.

Second, stem selection is an extremely important part of the procedure. The conventional cementless femoral component we used in both surgeries has been classified as a fit and fill type and is designed for the purpose of increasing the intramedullary occupancy rate of the femur and increasing the initial fixation [16]. Since the greater trochanter is positioned distally, it does not have the shape of the normal intertrochanteric crest; therefore, securing stability by using the proximal metaphyseal fit type component is considered difficult.

In both cases, postoperative abduction contracture occurred and the patients developed LLD. In addition to extension of the leg length, it is thought that this is due to the decrease in soft tissue flexibility, including that of the gluteus medius muscle, due to the scar tissue that is often formed around the gluteus medius following DTT. Fortunately, abduction contracture in both patients improved significantly within two months under physical therapy, and the final clinical results were excellent.

Although both patients were allowed to stand and walk with full weight bearing from the early postoperative period, the final radiographs did not show subsidence or loosening. Therefore, it seems that the stem used in both cases can be considered appropriate for THA following DTT.

We herein described two cases of THA following DTT. Although THA was needed to have more attention during operations, it has become clear that it is a safe and effective treatment for hip osteoarthritis following DTT. Furthermore, it was suggested that postoperative abductor contracture was more likely than usual THA, but the abductor contracture was reversible.

\section{Conflicts of Interest}

The authors declare that they have no conflicts of interest.

\section{References}

[1] K. Takata, S. Maniwa, and M. Ochi, "Surgical treatment of high-standing greater trochanter," Archives of orthopaedic and trauma surgery, vol. 119, no. 7-8, pp. 461-463, 1999.

[2] A. S. Kelikian, M. O. Tachdjian, M. J. Askew, and M. Jasty, "Greater trochanteric advancement of the proximal femur: a clinical and biomechanical study," The Hip, vol. 77, 1983.

[3] D. Schneidmueller, C. Carstens, and M. Thomsen, "Surgical treatment of overgrowth of the greater trochanter in children and adolescents," Journal of Pediatric Orthopaedics, vol. 26, no. 4, pp. 486-490, 2006.

[4] P. M. Stevens and S. S. Coleman, "Coxa Breva," Journal of Pediatric Orthopaedics, vol. 5, no. 5, pp. 515-521, 1985.

[5] B. Joseph, G. Varghese, K. Mulpuri, K. L. Narasimha Rao, and N. S. Nair, "Natural evolution of Perthes disease: a study of 610 children under 12 years of age at disease onset," Journal of Pediatric Orthopaedics, vol. 23, no. 5, pp. 590-600, 2003.

[6] N. Shohat, R. Gilat, R. Shitrit, Y. Smorgick, Y. Beer, and G. Agar, "A long-term follow-up study of the clinical and radiographic outcome of distal trochanteric transfer in LeggCalvé-Perthes' disease following varus derotational osteotomy," The bone \& joint journal, vol. 99-B, no. 7, pp. 987992, 2017.

[7] F. L. Garcia, C. H. F. Picado, and L. P. Cardinali, "Does trochanteric transfer eliminate the Trendelenburg sign in adults?" Clinical orthopaedics and related research, vol. 469, no. 7, pp. 1984-1990, 2011.

[8] V. A. Papavasiliou and J. M. Kirkos, "Reconstruction of residual deformities of the hip. A new surgical option," Clinical Orthopaedics and Related Research, vol. 341, pp. 123-127, 1997.

[9] U. Givon, A. Schindler, A. Ganel, and O. Levy, "Distal transfer of the greater trochanter revisited: long-term follow-up of nine hips," Journal of Pediatric Orthopaedics, vol. 15, no. 3, pp. 346348, 1995.

[10] M. F. Macnicol and D. Makris, "Distal transfer of the greater trochanter," The Journal of Bone and Joint Surgery. British, vol. 73-B, no. 5, pp. 838-841, 1991.

[11] M. Sonohata, M. Kitajima, S. Kawano, R. Tanaka, and M. Mawatari, "Total hip arthroplasty with femoral subtrochanteric osteotomy after Schanz osteotomy," Journal of Orthopaedic Science, vol. 21, no. 4, pp. 469-474, 2016.

[12] M. Sonohata, M. Kitajima, S. Kawano, and M. Mawatari, "Comparison of total hip arthroplasty outcomes after failed femoral wedge or curved varus osteotomy," The Open Orthopaedics Journal, vol. 25, no. 12, pp. 208-217, 2018.

[13] M. Kuribayashi, K. A. Takahashi, M. Fujioka, K. Ueshima, S. Inoue, and T. Kubo, "Reliability and validity of the Japanese Orthopaedic Association hip score," Journal of Orthopaedic Science, vol. 15, no. 4, pp. 452-458, 2010.

[14] T. Utsunomiya, G. Motomura, S. Ikemura, S. Hamai, J.i. Fukushi, and Y. Nakashima, "The results of total hip arthroplasty after Sugioka transtrochanteric anterior rotational osteotomy for osteonecrosis," The Journal of Arthroplasty, vol. 32, no. 9, pp. 2768-2773, 2017.

[15] T. Iwase, Y. Hasegawa, S. Iwasada, S. Kitamura, and H. Iwata, "Total hip arthroplasty after failed intertrochanteric valgus osteotomy for advanced osteoarthrosis," Clinical Orthopaedics and Related Research, vol. 364, pp. 175-181, 1999.

[16] M. Sonohata, M. Kitajima, S. Kawano, and M. Mawatari, "Wear of XLPE liner against zirconium heads in cementless total hip arthroplasty for patients under 40 years of age," Hip International, vol. 27, no. 6, pp. 532-536, 2017. 